\title{
RECURSIVE CALCULATION OF THE NET PREMIUM FOR LARGEST CLAIMS REINSURANCE COVERS
}

\author{
E. KREMER
}

\section{Universität Hamburg}

\begin{abstract}
In the present paper the author investigates the problem of calculating the net premium for some versions of the largest claims reinsurance cover. A very handy recursive rating method is derived by applying some recursion formulas for the expectations of order statistics.
\end{abstract}

\section{INTRODUCTION}

The calculation of the exact premium for an insurance treaty is a very old and fundamental problem in nonproportional reinsurance. Mathematical rating methods for the classical stop-loss and excess-of-loss treaties are already presented by Helbig (1953) and Ammeter (1955). An alternative to the excess-of-loss cover, the so-called ECOMOR-treaty was investigated by the French actuary THÉPAUT (1950), whereas the more familiar largest claims reinsurance treaty came to a mathematical treatment not earlier than in 1964 (AMMETER 1964). Some few years ago the author of the present paper took up again the problem of premium calculation for the last two treaties, inspired by a paper of BENKTANDER (1978). In a sequence of seven papers (KREMER 1982, 1983, 1984a, $b$, $1985 a, b)$ refined and generalized results were presented for those covers by applying results from the theory of order statistics. General asymptotic premium formulas were proved (see KREMER 1982, 1983, 1984b), as well as more specialized finite statements (see KREMER 1985a, b). In the present paper the calculation of the net premium is reinvestigated for a generalized largest claims cover and a theorem is proved, allowing a simple recursive premium calculation. The present paper is in some sense a counterpart to a previous one by PANJER (1981), stating a method for recursive calculating the net premium of a stop-loss-treaty.

\section{THE REINSURANCE COVER}

Consider a collective $K$ of risks and let $N$ denote the random variable of the number of claims. The corresponding claim amounts are described by the random variables $X_{1}, X_{2}, \ldots, X_{N}$. Denote by

$$
X_{N_{1}} \leqslant \cdots \leqslant X_{N N}
$$

the claims ordered in increasing size. Furthermore let $c_{1}, c_{2}, c_{3}, \ldots, c_{p}$ be real constants and define for a given natural number $p$

$$
R_{N}\left(c_{1}, \ldots, c_{p}\right)=\sum_{i=1}^{p} c_{i} \cdot X_{N N-i+1},
$$

ASTIN BULLETIN Vol 16, No 2 
(we make the convention that $X_{N-1+1}=0$ if $N<i$ ) describing the amount paid by the reinsurer. Consequently a vector $\left(c_{1}, \ldots, c_{p}\right)$ defines a reinsurance treaty, we call it generalized largest claims cover (in short: $\operatorname{LCR}\left(c_{1}, \ldots, c_{p}\right)$ ).

In the special cases:

(a) $c_{1}=c_{2}=\cdots=c_{p}=1$ we get the classical largest claims treaty, covering the $p$ largest claims.

(b) $c_{1}=c_{2}=\cdots=c_{p-1}=1, c_{p}=(1-p)$ we get the classical ECOMOR treaty, covering all claims in excess of the $p$ th largest claim.

Define by

$$
\mu_{p}:=\mu\left(c_{1}, c_{2}, \ldots, c_{p}\right):=E\left[R_{N}\left(c_{1}, c_{2}, \ldots, c_{p}\right)\right]
$$

the net premium of the $\operatorname{LCR}\left(c_{1}, \ldots, c_{p}\right)$-treaty. Assuming the claim sizes $X_{1}, X_{2}, X_{3}, \ldots$ to be i.i.d. with continuous distribution function and independent of $N$ then one has (see KREMER 1985a)

$$
\begin{aligned}
\mu_{p} & =\sum_{t=1}^{p} c_{t} E\left(X_{N N-i+1}\right) \\
& =\sum_{i=1}^{p} \frac{c_{t}}{\Gamma(i)} \int_{0}^{1} F^{-1}(t)(1-t)^{t-1} M^{(t)}(t) d t,
\end{aligned}
$$

where:

$$
F^{-1}(u):=\inf \{x: F(x) \geqslant u\}
$$

is the pseudo-inverse of the claim size distribution function and $M^{(i)}$ is the $i$ th derivative of the moment generating function

$$
M(s)=\sum_{n=0}^{\infty} P(N=n) \cdot s^{n}
$$

of the claims number $N$. So from the theoretical point of view there is no problem in calculating the net premium $\mu_{p}$. But from the empirical point of view the above result is not satisfying, since one has to estimate $F$ as well as $M^{(1)}(s), i=1, \ldots, p$ from known past claim sizes and claim numbers. This led to the development of different handier approaches for estimating $\mu_{p}$. Besides directly estimating $\mu_{p}$ by statistical methods, especially by the so called burning cost procedures, actuaries thought of deriving simple upper bounds for $\mu_{p}$ (see BENKTANDER 1978, KREMER 1983) which become asymptotically exact (see KREMER 1982). In the following section we present a different approach, which allows a simple recursive premium calculation.

\section{A RECURSIVE RATING PROCEDURE}

We consider a family of claim number distributions satisfying the recursion

$$
p_{n}=p_{n-1}(a+b / n), \quad m=1,2,3, \ldots, N
$$

where

$$
p_{n}=P(N=n)
$$


Special cases are the Poisson distribution, the binomial distribution, the negative binomial distribution and the geometric distribution (see PANJER 1981). A characterization of the distributions following the above recursion is given in SUNDT and JEWELL (1981).

A first step for deriving a recursive premium calculation method might be seen in the simple equation:

$$
\mu_{p}=c_{p} E\left(X_{N N-p+1}\right)+\mu_{p-1} .
$$

But this leads to nothing new than to formula (2.1). A better approach is given in the following basic result, giving a higher order recursion:

Theorem. Assume the claim sizes to be i.i.d. and the claims number probabilities $p_{n}$ to satisfy the above recursion. Then one has for the net premiums $\mu_{p}, \mu_{p-1}, \mu_{p-2}$ of treaties $\operatorname{LCR}\left(c_{1}, \ldots, c_{p}\right), \operatorname{LCR}\left(c_{1}, \ldots, c_{p-1}\right), \operatorname{LCR}\left(c_{1}, \ldots, c_{p-2}\right)$ the recursion

$$
\begin{aligned}
\mu_{p}= & \mu_{p-1}\left(1+\frac{c_{p}}{c_{p-1}} \frac{p-1+a+b}{p-1}\right)-\mu_{p-2} \frac{c_{p}}{c_{p-1}} \frac{p-1+a+b}{p-1} \\
& +E\left(N \cdot X_{N N-p+2}\right) \frac{a-1}{p-1} c_{p}
\end{aligned}
$$

starting with

$$
\mu_{1}=c_{1} \mu_{1}^{*}, \quad \mu_{2}=c_{2} \mu_{2}^{*}+\mu_{1}
$$

$\mu_{1}^{*}$ denoting the mean value of the ith largest claim:

$$
\mu_{1}^{*}=E\left(X_{N N}\right), \quad \mu_{2}^{*}=E\left(X_{N N-1}\right) .
$$

The proof of the theorem is based on the following

LEMMA. Under the assumptions of the theorem one has:

$$
E\left(X_{N N-p+1}\right)=\frac{p-1+a+b}{p-1} E\left(X_{N N-p+2}\right)+\frac{a-1}{p-1} E\left(N \cdot X_{N N-p+2}\right) .
$$

Proof. Denote by

$$
\nu_{n n-k}:=E\left(X_{n n-k}\right)
$$

for fixed claim number $N=n$. One gets from a well-known recursion (see DAvID 1980 , p. 46 with $r=n-p+1)$

$$
(p-1) \nu_{n-p+1}+(n-p+1) \nu_{n n-p+2}=n \cdot \nu_{n-1 n-p+1},
$$

implying by multiplying with $p_{n}$, summing $n$ from $p$ up to $\infty$ and substituting $p_{n}=p_{n-1}(a+b / n)$

$$
\begin{aligned}
(p-1) \sum_{n=p}^{\infty} p_{n} \nu_{n} n-p+1 & =-\sum_{n=p-1}^{\infty}(n-p+1) p_{n} \nu_{n n-p+2} \\
& +a \sum_{n=p-1}^{\infty} n p_{n} \nu_{n n-p+2} \\
& +(a+b) \sum_{n=p-1}^{\infty} p_{n} \nu_{n n-p+2}
\end{aligned}
$$


This means:

$$
\begin{aligned}
(p-1) E\left(X_{N N-p+1}\right)= & -E\left((N-(p-1)) X_{N N-p+2}\right) \\
& +a E\left(N \cdot X_{N N-p+2}\right)+(a+b) E\left(X_{N N-p+2}\right)
\end{aligned}
$$

and finally:

$$
E\left(X_{N N-p+1}\right)=\frac{p-1+a+b}{p-1} E\left(X_{N N-p+2}\right)+\frac{a-1}{p-1} E\left(N \cdot X_{N N-p+2}\right)
$$

Proof of the Theorem. Since

$$
\begin{gathered}
\mu_{p}=c_{p} E\left(X_{N N-p+1}\right)+\mu_{p-1} \\
E\left(X_{N N-p+2}\right)=\frac{1}{c_{p-1}} \cdot\left(\mu_{p-1}-\mu_{p-2}\right)
\end{gathered}
$$

the statement follows at once from the Lemma.

The above recursion is not very practicable due to the term $E\left(N \cdot X_{N N-p+2}\right)$ which is unknown. Nevertheless this problem can partly be overcome by the following:

Statement. Consider a sequence $\left(K_{k}, k \geqslant 1\right)$ of collectives $K_{k}$ with claims number $N_{k}$ satisfying

$$
\lim _{k \rightarrow \infty} E\left(N_{k}\right)=\infty, \quad \lim _{k \rightarrow \infty} \frac{\sqrt{\operatorname{Var}\left(N_{k}\right)}}{E\left(N_{k}\right)}=0 .
$$

For the i.i.d. claim-sizes $X_{1}$ we assume

$$
0<d_{1} \leqslant\left|X_{1}\right| \leqslant d_{2}, \quad \forall i
$$

and for $p=p_{k}$ depending on $k$

$$
\lim _{k \rightarrow \infty} \frac{p_{k}}{E\left(N_{k}\right)}=s \in(0,1) .
$$

Then

$$
\lim _{k \rightarrow \infty} \frac{E\left(N_{k} X_{N_{k} N_{h}-p_{h}+2}\right)}{E\left(N_{k}\right) E\left(X_{N_{k} N_{k}-p_{k}+2}\right)}=1
$$

Proof. One has without loss of generality

$$
\begin{aligned}
N_{k} \rightarrow \infty & \text { almost surely } \\
\frac{N_{k}}{E(N k)} \rightarrow 1 & \text { almost surely } \\
\frac{X_{N_{h} N_{h}-p_{h}+2}}{E\left(X_{N_{k} N_{h}-p_{k}+2}\right)} \rightarrow 1 & \text { almost surely, }
\end{aligned}
$$


since

$$
\begin{aligned}
X_{N_{k} N_{k}-p_{k}+2} & \rightarrow F^{-1}(1-s) \quad \text { almost surely } \\
E\left(X_{N_{k} N_{k}-p_{k}+2}\right) & \rightarrow F^{-1}(1-s)
\end{aligned}
$$

(compare KREMER 1982, p. 51). This implies the result with a version of Lebesgue's dominated convergence theorem.

According to this result one tries to approximate

$$
E\left(N X_{N N-p+2}\right)
$$

by

$$
\lambda E\left(X_{N N-p+2}\right)=\lambda \frac{1}{c_{p-1}}\left(\mu_{p-1}-\mu_{p-2}\right)
$$

with

$$
\lambda=E(N)=\frac{a+b}{1-a}
$$

(see JOHNSON and KOTZ 1969, p. 37) resulting in the approximate recursion:

$$
\begin{gathered}
\mu_{p}=\mu_{p-1}\left(1+K_{p}\right)-\mu_{p-2} K_{p} \\
\quad \text { where } \\
K_{p}:=\frac{c_{p}}{c_{p-1}} .
\end{gathered}
$$

REMARK. (1) Denote by

$$
\mu_{(k)}=E\left(N_{k}\right) E\left(X_{l}\right)
$$

the total claims amount. Then the above statement implies:

$$
\lim _{k \rightarrow \infty}\left(\frac{E\left(N_{k} X_{N_{k} N_{k}-p_{k}+2}\right)}{\mu_{(k)}}-\frac{E\left(N_{k}\right) E\left(X_{N_{k} N_{k}-p_{k}+2}\right)}{\mu_{(k)}}\right)=0 .
$$

Consequently the approximations (3.3) are mainly suited for calculating the premium rates:

$$
\rho_{p}^{(k)}=\frac{\mu_{p}}{\mu_{(k)}} .
$$

(2) It can easily be shown that $N$ and $X_{N N-p+2}$ are positively correlated. Hence the recursion (3.3) implies an upper bound in the case, where $c_{1}, \ldots, c_{p}$ are positive. In particular this condition is fulfilled for the classical largest claims treaty. 


\section{ExAMPLE}

Suppose $a, b$ are arbitrary, but that

$$
c_{p}=c_{p-1},
$$

implying the approximate recursion (3.3) for the general treaty

$$
\mu_{p} \cong 2 \mu_{p-1}-\mu_{p-2} \text {, }
$$

which is very attractive from the practical point of view.

Let us compare this recursion with the premiums calculated by $(2.1)$ in the case $\lambda=100$ and

(a) For a Pareto claim size distribution function:

$$
F(x)=1-x^{-a}, \quad x \geqslant 1 .
$$

Denote by $\mu_{p}$ the net premium calculated by the formula

$$
\begin{aligned}
\hat{\mu}_{p} & =\lambda^{1 / \alpha} \frac{\alpha}{\alpha-1} \frac{\Gamma(p+1-1 / \alpha)}{\Gamma(p)} \\
\Gamma(y) & =\int_{0}^{\infty} n^{y-1} \exp (-n) d n,
\end{aligned}
$$

which is known to be a very good approximation to $\mu_{p}$ (see AMMETER 1964). Finally define by $\hat{\hat{\mu}}_{p}$ the net premium calculated by the above recursion. With the mean total claims amount

$$
\mu=E(N) \frac{\alpha}{\alpha-1}
$$

one gets the premium rates

$$
\hat{\rho}_{p}=\hat{\mu}_{p} / \mu, \quad \hat{\hat{\rho}}_{p}=\hat{\hat{\mu}}_{p} / \mu .
$$

(a.1) $\alpha=2.0$

\begin{tabular}{c|cccccc} 
& $p=$ & 1 & 2 & 3 & 4 & 5 \\
\hline$\hat{\rho}_{p}$ & & $8.9 \%$ & $13.3 \%$ & $16.6 \%$ & $19.4 \%$ & $21.8 \%$ \\
$\hat{\hat{\rho}}_{p}$ & & $8.9 \%$ & $13.3 \%$ & $17.7 \%$ & $19.9 \%$ & $22.2 \%$ \\
& $p=$ & 6 & 7 & 8 & 9 & 10 \\
\hline$\hat{\rho}_{p}$ & & $24 \%$ & $26.0 \%$ & $27.8 \%$ & $29.6 \%$ & $31.2 \%$ \\
$\hat{\hat{\rho}}_{p}$ & & $24.2 \%$ & $26.2 \%$ & $28.0 \%$ & $29.7 \%$ & $31.3 \%$
\end{tabular}

(a.2) $\alpha=3.0$ 
LARGEST CLAIMS REINSURANCE COVERS

\begin{tabular}{c|cccccc} 
& $p=$ & 1 & 2 & 3 & 4 & 5 \\
\hline$\hat{\rho}_{p}$ & & $4.2 \%$ & $7.0 \%$ & $9.3 \%$ & $11.4 \%$ & $13.3 \%$ \\
$\hat{\rho}_{p}$ & & $4.2 \%$ & $7.0 \%$ & $9.8 \%$ & $11.6 \%$ & $13.4 \%$ \\
& $p=$ & 6 & 7 & 8 & 9 & 10 \\
\hline$\hat{\rho}_{p}$ & & $15.1 \%$ & $16.7 \%$ & $18.3 \%$ & $19.8 \%$ & $21.3 \%$ \\
$\hat{\hat{\rho}}_{p}$ & & $15.2 \%$ & $16.8 \%$ & $18.4 \%$ & $19.9 \%$ & $21.4 \%$
\end{tabular}

(b) For an exponential claims size distribution function

$$
F(x)=1-\exp (-(\alpha-1)(x-1)), \quad x \geqslant 1 .
$$

Denote by $\hat{\mu}_{p}$ the net premium calculated by the formula:

$$
\hat{\mu}_{p}=\frac{p \alpha-1}{p-1}+\frac{p}{\alpha-1} \sum_{j=p}^{\infty} \frac{1}{j}\left(1-\sum_{n=0}^{j-1} \operatorname{Prob}(N=n)\right)
$$

(see KREMER 1986) and by $\hat{\hat{\mu}}_{p}$ the above recursively calculated net premium (see $(4.1)$ ). The premium rates $\hat{\rho}_{p}$ and $\hat{\hat{\rho}}_{p}$ are defined as above.

(b.1) $\alpha=2.0$

\begin{tabular}{c|cccccc} 
& $p=1$ & 1 & 2 & 3 & 4 & 5 \\
\hline$\hat{\rho}_{p}$ & & $3.1 \%$ & $5.7 \%$ & $8.0 \%$ & $10.2 \%$ & $12.2 \%$ \\
$\hat{\hat{\rho}}_{p}$ & & $3.1 \%$ & $5.7 \%$ & $8.3 \%$ & $10.4 \%$ & $12.4 \%$ \\
& $p=$ & 6 & 7 & 8 & 9 & 10 \\
\hline$\hat{\rho}_{p}$ & & $14.2 \%$ & $16.1 \%$ & $17.9 \%$ & $19.6 \%$ & $21.3 \%$ \\
$\hat{\hat{\rho}}_{p}$ & & $14.3 \%$ & $16.2 \%$ & $17.9 \%$ & $19.7 \%$ & $21.3 \%$
\end{tabular}

(b.2) $\alpha=3.0$

\begin{tabular}{c|cccccc} 
& $p=$ & 1 & 2 & 3 & 4 & 5 \\
\hline$\hat{\rho}_{p}$ & & $2.4 \%$ & $4.5 \%$ & $6.4 \%$ & $8.1 \%$ & $9.8 \%$ \\
$\hat{\hat{\rho}}_{p}$ & & $2.4 \%$ & $4.5 \%$ & $6.5 \%$ & $8.2 \%$ & $9.9 \%$ \\
& $p=$ & 6 & 7 & 8 & 9 & 10 \\
\hline$\hat{\mu}_{p}$ & & $11.5 \%$ & $13.0 \%$ & $14.6 \%$ & $16.1 \%$ & $17.5 \%$ \\
$\hat{\hat{\mu}}_{p}$ & & $11.5 \%$ & $13.1 \%$ & $14.6 \%$ & $16.1 \%$ & $17.6 \%$
\end{tabular}

According to these numerical results the above recursion (4.1) can fully be recommended for practical premium rating. Notice that this gives a very simple and quick method to estimate the premiums of a classical largest claims cover 
with $p>2$, knowing only estimates for the expectations of the first and second largest claims of a portfolio of risks. In both examples the starting values can be calculated by the results in AMMETER (1964).

The example indicates that the approximations resulting from the statement are fairly good. They are upper bounds to the correct premium (cf. remark (2) of Section 3).

\section{ACKNOWLEDGEMENT}

The author is grateful to the referee for suggesting improvements to the paper.

\section{REFERENCES}

Ammeter, H. (1955) The Calculation of Premium Rates for Excess of Loss and Stop Loss Reinsurance Treaties. Nonproportional Reinsurance. Bruxelles, pp. 79-109.

AMmETER, H. (1964) The Rating of "Largest Claim" Reinsurance Covers. Quarterly letter from the Allgemeene Reinsurance Companies, Jubilee number 2, pp. 5-17.

BenkTANDER, G. (1978) Largest Claims Reinsurance (LCR): A Quick Method to Calculate LCRRisk Rates from Excess of Loss Risk Rates. ASTIN Bulletin, 54-58.

David, H. A. (1980) Order statistics. John Wiley, New York.

Helbig, M. (1953) Mathematische Grundlagen der Schadenexzedenten-rückversicherung. Festschrift für Emil Bebler, 189-197.

JoHNSON, N. I. and KoTZ, S. (1969) Discrete distributions. John Wiley, New York.

KREMER, E. (1982) Rating of Largest Claims and ECOMOR Reinsurance Treatise for Large Portfolios. ASTIN Bulletin, 47-56.

Kremer, E. (1983) Distribution-Free Upper Bounds on the Premiums of the LCR and ECOMOR Treaties. Insurance: Mathematics and Economics, 209-213.

KREMER, E. (1984a) Rating of Nonproportional Reinsurance Treatise Based on Ordered Claims. Premium Calculation in Insurance. Proceedings of the NATO Advanced Study Institute, Leuven, pp. 285-314.

Kremer, E. (1984b) An Asymptotic Formula for the Net-Premium of some Reinsurance Treaties. Scandinavian Actuarial Journal, 11-22.

Kremer, E. (1985a) Finite Formulae for the Premium of the General Reinsurance Treaty Based on Ordered Claims. Insurance: Mathematics \& Economics, 233-238.

Kremer, E. (1985b) Reinsurance Premiums Under Special Claims Number Distribution. Submitted for publication.

Kremer, E. (1986) Simple Formulas for the Net Premiums of the LCR and ECOMOR Treaties Under Exponential Claims Sizes. Blätter der Deutschen Gesellschaft für Versicherungs mathematik.

SUNDT, B. and JEWELL, W. S. (1981) Further Results on Recursive Evaluation of Compound Distributions. ASTIN Bulletin, 27-30.

PANJer, H. H. (1981) Recursive Evaluation of a Family of Compound Distributions. ASTIN Bulletin, 22-26.

Thépaut, A. (1950) Le Traité d'Excédent du Coût relativ., Bulletin Trimestriel de L'Institut des Actuaries Francais, No. 2.

Prof. Dr. E. KREMER

Institut für Mathematische Statistik, Universität Hamburg, D-2 Hamburg 13. 